\title{
Обработка технико-экономической информации в системе геотехнического мониторинга
}

\author{
Н.В. Дорофеев, Е.С. Панькина, А.В. Греченева, Р.В. Романов \\ Владимирский государственный университет им. А.Г. и Н.Г. Столетовых, \\ 600000, г. Владимир, ул. Горького, 87, Российская Федерация \\ E-mail: dorofeevnv@yandex.ru,pankina@bsu.edu.ru,grechenevaav@yandex.ru, \\ romanov.roman.5@yandex.ru
}

\begin{abstract}
Аннотация
В данной статье предлагается метод обработки параметров геотехнической системы, предназначенный для повышения эффективности функционирования систем геотехнического мониторинга в условиях риска нарушения геодинамической устойчивости и технико-экономических ограничений при организации мониторинговых работ. Предлагаемый метод позволяет подобрать технические параметры системы мониторинга и выбрать точки мониторинга в геотехнической системе на основе экономических показателей. В качестве критерия отбора выступает критерий минимизации затрат на внедрение системы геотехнического мониторинга относительно возможного ущерба, который выражается в денежном эквиваленте, в случае нарушения устойчивости геотехнической системы или еe анализируемого участка. Затраты на внедрение системы геотехнического мониторинга определяются исходя из её стоимости, постоянных затрат на её обслуживание, а также технических параметров. Среди технических параметров, определяющих затраты на внедрение системы геотехнического мониторинга, выделяются затраты в случае пропуска деструктивных геотехнических процессов, ложного срабатывания системы, затрат на регистрацию одного параметра геотехнической системы в одной точке определенным методом. Для снижения количества контролируемых параметров и точек измерения предлагается проводить измерения в ключевых точках контроля, а выбор контролируемых параметров осуществляется на основе бифуркационного подхода. Практическая проверка разработанного метода обработки параметров геотехнической системы проводилась при обнаружении суффозионных процессов в условиях городской застройки в городе Муроме Владимирской области Российской Федерации. Предлагаемый метод позволил существенно сократить затраты на проведение мониторинговых работ с сохранением точности работы системы мониторинга.
\end{abstract}

Ключевые слова: геотехническая система, системы мониторинга, технико-экономический анализ, оптимизация, бифуркационные параметры.

Благодарности: работа выполнена при финансовой поддержке гранта Президента Российской Федерации № МД-1800.2020.8.

Для цитирования: Дорофеев Н.В., Панькина Е.С., Греченева А.В., Романов Р.В. 2020. Обработка технико-экономической информации в системе геотехнического мониторинга. Экономика. Информатика. 47 (3): 638-647. DOI 10.18413/2687-0932-2020-47-3-638-647.

\section{Processing of technical and economic information in the system of geotechnical monitoring}

\author{
N.V. Dorofeev, E.S. Pankina, A.V. Grecheneva, R.V. Romanov \\ Vladimir State University, \\ 600000, Vladimir, St. Gorkogo, 87, Russian Federation \\ E-mail: dorofeevnv@yandex.ru, pankina@bsu.edu.ru,grechenevaav@yandex.ru, \\ romanov.roman.5@yandex.ru
}

\begin{abstract}
This article proposes a method for processing the parameters of a geotechnical system, designed to increase the efficiency of the functioning of geotechnical monitoring systems under conditions of the risk of violation of geodynamic stability and technical and economic limitations when organizing monitoring works. The
\end{abstract}


proposed method allows you to select the technical parameters of the monitoring system and select monitoring points in the geotechnical system based on economic indicators. The selection criterion is the criterion of minimizing the cost of introducing a geotechnical monitoring system with respect to possible damage, which is expressed in monetary terms, in case of a violation of the stability of the geotechnical system or its analyzed area. The costs of implementing a geotechnical monitoring system are determined based on its cost, fixed costs of its maintenance, as well as technical parameters. Among the technical parameters that determine the costs of implementing a geotechnical monitoring system, there are costs in the event of missing destructive geotechnical processes, false alarms of the system, costs of registering one parameter of a geotechnical system at one point using a specific method. To reduce the number of monitored parameters and measurement points, it is proposed to carry out measurements at key control points, and the choice of monitored parameters is based on the bifurcation approach. Practical verification of the developed method for processing the parameters of the geotechnical system was carried out when suffusion processes were detected in urban development in the city of Murom, Vladimir region, Russian Federation. The proposed method made it possible to significantly reduce the costs of monitoring work while maintaining the accuracy of the monitoring system.

Keywords: geotechnical system, monitoring systems, technical and economic analysis, optimization, bifurcation parameters.

Acknowledgements: This paper is an output of the science project executed with the support of a grant of the President of the Russian Federation No. MD-1800.2020.8.

For citation: Dorofeev N.V., Pankina E.S., Grecheneva A.V., Romanov R.V. 2020. Processing of technical and economic information in the system of geotechnical monitoring. Economics. Information technologies. 47 (3): 638-647 (in Russian). DOI 10.18413/2687-0932-2020-47-3-638-647.

\section{Введение}

Развитие техносферы сопровождается изменением количества и силы взаимосвязей между компонентами природной и технической среды. Изменения, которые негативно сказываются на устойчивости геотехнических систем, приводят к авариям и катастрофам различного масштаба [Иноземцев, Редков, 2017; Теличенко и др., 2006; Сосунов, 2010]. Системы мониторинга состояния геотехнических систем строятся на двух основных подходах. Один подход заключается в организации наблюдений за изменениями в геологической среде, выделении и прогнозировании начальной стадии развития опасных геодинамических процессов. В этом подходе учитываются гидрологические, геологические, климатические и другие параметры [Квартальнов, Макулов 2017; Осокин и др., 2014]. Другой подход заключается в наблюдении за технико-эксплуатационными параметрами сооружений, выявлении и прогнозировании деструктивных процессов и оценки параметров устойчивости сооружений [Vasilyev et al., 2018; Kostarev et al., 2013; Bondarik, 2012]. Несмотря на достоинства и недостатки обоих подходов на практике, всегда возникает ряд технических, организационных и экономических вопросов. Например, какие параметры геотехнической системы необходимо регистрировать, какими техническими средствами необходимо регистрировать выбранные параметры, каким образом размещать технические средства и осуществлять сбор данных, какое количество датчиков необходимо и в каких местах необходимо измерять, какие затраты будут произведены, в чем будет заключаться выгода и так далее [ГОСТ Р 53778-2010; Улицкий, Шашкин, 1999; Мальцев и др., 2014].

Целью данной работы является повышение эффективности систем геотехнического мониторинга в условиях риска нарушения геодинамической устойчивости и экономических ограничений на организацию мониторинговых работ за счет разработки метода обработки параметров геотехнической системы на основе данных технико-экономического анализа.

\section{Методы и подходы}

Экономическая эффективность применения системы геотехнического мониторинга достигается при снижении ущерба от негативного развития процессов в геотехнической системе за счет снижения погрешности измерений и вероятности ложного обнаружения 
предкризисных ситуаций в геотехнической системе, повышении вероятности правильного обнаружения предкризисных ситуаций и их прогнозирования на более ранней стадии [Леонов, Шкаруба, 2012]. Таким образом, экономическая эффективность внедрения системы геотехнического мониторинга зависит от метрологического, методологического и алгоритмического обеспечения последней.

Следует отметить, что в случае организации мониторинговых работ на базе системы мониторинга одного типа в различных геотехнических системах экономическая эффективность от внедрения будет отличаться, причем различие может составлять несколько порядков. Это связано с индивидуальными особенностями функционирования геотехнических систем и протекающих в них процессов, которые (индивидуальные особенности) не отражаются или отражаются не в полной мере в функционировании систем геотехнического мониторинга. Как правило, системы геотехнического мониторинга разрабатываются под конкретный объект (геотехническую систему) и обладают низкой адаптивностью к качественным и количественным изменениям в геотехнической системе [Kuzichkin et al., 2018a].

Составление карты экономических ущербов с разделением на зоны значимости и объединением компонентов геотехнической системы по объектам различных уровней (местного, локального, регионального). Для каждой зоны определяется возможный экономический ущерб и значимость данной зоны в функционировании геотехнической системы с учетом возможного социального критерия (1) и (2). Кластеризация на зоны карты осуществляется на основе алгоритма расширяющегося нейронного газа [Sledge, Keller, 2008].

$$
C_{G D}=\sum_{i=1}^{n} C_{i D}
$$

где $C_{G D}$ - общий экономический ущерб в геотехнической системе; $C_{i D}$ - экономический ущерб отдельной зоны геотехнической системы; n - количество зон;

$$
C_{i D}=\sum_{j=1}^{m}\left(C_{j}\left(W_{j}\right) P_{j} r_{j}+S_{j}\left(r_{j}\right)\right)
$$

где $C_{j}$ - значимость $j$-го компонента в $i$-й зоне в зависимости от его износа $W_{j}, P_{j}$ - стоимость $j$-го компонента в $i$-й зоне; $r_{j}$ - риск (вероятность) разрушения, характеризующее возможную степень разрушения, при этом 0 - отсутствие разрушений, 1 - полное разрушение; $S_{j}$ - социальный ущерб, выраженный в денежном эквиваленте.

Рассчитанный возможный экономический ущерб, в случае нарушения функционирования каждой зоны, является определяющим критерием при внедрении системы геотехнического мониторинга. Стоимость внедрения системы мониторинга должна быть ниже возможного экономического ущерба, при этом затраты на обслуживание системы геотехнического мониторинга в течение определенного времени задаются исходя из её технических параметров:

$$
C_{D G} \geq C_{I}=C_{S}+\sum_{t=0}^{\Delta}\left(C_{M}+k C_{S}\left(1-e^{-t / T_{0}}\right)\right)+\sum_{p} \sum_{b}\left(p_{1 \rightarrow 0} C_{p}+p_{0 \rightarrow 1} C_{S M}\right),
$$

где $C_{I}$ - стоимость внедрения системы геотехнического мониторинга; $C_{S}-$ стоимость системы геотехнического мониторинга; $t$ - время эксплуатации системы мониторинга, $\Delta-$ разрешенный период внедрения системы геотехнического мониторинга; $C_{M}$ - постоянные затраты на обслуживание системы геотехнического мониторинга; $k-$ поправочный коэффициент в диапазоне от 1,1 до 1,$4 ; T_{0}$ - средняя наработка на отказ в системе геотехнического мониторинга; $p$ - неблагоприятный фактор (анализируемые параметры геотехнической системы); $\mathrm{b}$ - точки бифуркации для каждого параметра $p ; p_{1 \rightarrow 0}-$ вероятность пропуска системой геодинамического мониторинга перехода параметра р в точку бифуркации $\mathrm{b} ; C_{P}-$ ущерб в случае пропуска неблагоприятного события $p ; p_{0 \rightarrow 1}-$ вероятность ложного срабатывания системы геодинамического мониторинга при обнаружении перехода параметра 
$\mathrm{p}$ в точку бифуркации $\mathrm{b}$; $C_{S M}$ - стоимость произведенных работ по устранению неблагоприятного события $p$ в случае ложного срабатывания.

С учетом индивидуальных особенностей геотехнической системы к рассмотрению вопроса о внедрении принимается система геотехнического мониторинга, которая удовлетворяет следующим условиям:

$$
\min \left(C_{\cos t}\right), C_{\cos t}=\left\{\forall C_{I} \mid\left(C_{I} \leq C\right) \cap(\Delta \geq T)\right\},
$$

где $C$ - допустимые затраты на промежутке времени Т.

Сложный характер функционирования геотехнических систем описывается большим количеством разнородных параметров, некоторые из которых являются взаимозависимыми. Для оценки текущего состояния и прогнозирования устойчивости геотехнической системы необходимо контролировать изменение значений этих параметров на большой площади. Для сокращения контролируемых параметров и соответственно снижения стоимости внедрения системы геотехнического мониторинга предлагается определить параметры по степени зависимости друг от друга, степени влияния на устойчивость геотехнической системы и затрат на проведение измерений данных параметров. На основании модульного принципа построения формируется модель геотехнической системы или её отдельные участки [Grecheneva et al., 2018]. На основании данной модульной модели определяются параметры $p$, по которым оценивается устойчивость геотехнической системы St [Kuzichkin et al., 2018b]. На основании дисперсионного анализа оценивается степень влияния параметров $p$ на устойчивость геотехнической системы $S t_{1}$. В результате оценки получается вектор

$$
S t_{1}=\left\{f\left(p_{1}\right), \ldots, f\left(p_{n}\right)\right\},
$$

где $f\left(p_{i}\right)$ - степень влияния $i$-го параметра на устойчивость геотехнической системы.

По аналогии для всех параметров геотехнической системы составляется матрица $P_{i j}$ зависимости $i$-го параметра от $j$-го $f\left(p_{i}, p_{j}\right)$, такая, что

$$
P_{i j}=\left\{\begin{array}{l}
f\left(p_{i}, p_{j}\right), i \neq j \\
0, i=j
\end{array} .\right.
$$

На основании матрицы взаимных зависимостей параметров $P_{i j}$ составляются вектора устойчивости геотехнической системы $S t$ путем последовательной замены степеней влияния вектора $S t$ зависимых параметров $p$ на совокупность соответствующих параметров из строки вектора $P_{i j}$ для параметра $p$. Замена производится до тех пор, пока значения степеней влияния заменяющих параметров, при замене заменяемых, больше установленного порога $\varepsilon$.

Для всех параметров векторов St на основе диаграмм бифуркации по каждому параметру вектора определяются точки бифуркации [Petrochenko, Petrochenko, 2019; Иноземцев и др., 2010; Назаров, 2015], удаленность текущего состояния системы от этих точек, а так же степень значимости параметра. В качестве контроля выбираются параметры того вектора, который удовлетворяет следующим условиям:

$$
\min \left(\sum_{p} \frac{\left(\left|b_{p}-\bar{p}\right|-D\right) \Delta S K C_{M E} S t_{i}\{p\}}{D S}\right),
$$

где $\mathrm{p}$ - все элементы (параметры) вектора $S t_{i} ; b_{p}$ - значение бифуркационного параметра, при этом $b_{p}=\min \left(\left|b_{p i}-p(t)\right|\right), b_{p i}-\mathrm{i}$-я точка бифуркации для параметра $p ; \bar{p}$ - текущее среднее значение измеряемого параметра $p ; D$ - дисперсия измеряемого параметра $p ; \Delta S$ - площадь влияния параметра $p ; S$ - общая площадь анализируемого участка; $K$ - необходимое количество точек измерения параметра $p$ на площади $S ; C_{M E}-$ стоимость внедрения одной точки измерения; $S t_{i}\{p\}$ - степень влияния параметра р на устойчивость геотехнической системы. 
Параметр $C_{M E}$ в выражении (7) определяет стоимость измерения параметра конкретным методом, который выбирается исходя из технических и эксплуатационных возможностей применения в конкретной точке измерения. В случае возможности проводить измерения несколькими методами, выбирается метод, на внедрение которого (стоимость устанавливаемого оборудования и работ) требуется меньше затрат.

Уменьшить стоимость внедрения системы геодинамического контроля возможно, применив метод контроля, основанный на мониторинге устойчивости ключевых точек [Dorofeev et al, 2016]. В соответствии с этим методом контроль опасных процессов осуществляется в точках, которые наиболее чувствительны к развитию начальной фазы деструктивных процессов и проявлению их предвестников. Каждая ключевая тока контроля определяет общий тренд изменения параметров на некоторой площади. Повышенная чувствительность этих точек к изменениям в области контроля позволяет фиксировать негативные процессы на более ранних стадиях.

Следует отметить, что в начальный момент времени при проведении мониторинга устойчивости геотехнической системы апостериорные данные и зависимости между параметрами геотехнической системы могут отсутствовать. В этом случае строится робастная модель анализируемых участков геотехнической системы и на её основании определяются критические параметры и точки мониторинга. По мере накопления данных и выявления зависимостей модель уточняется, корректируется функционирование системы геотехнического мониторинга.

Таким образом, предлагаемый метод обработки параметров геотехнической системы базируется на результатах технико-экономического анализа и включает в себя следующие шаги:

1. Оценка рисков и возможных ущербов при нарушении устойчивости геотехнической системы и отдельных ее участков, а также классификации и выделении наиболее значимых зон мониторинга.

2. Формирование перечня измеряемых параметров.

3. Формирование модульной модели геотехнической системы или её анализируемого участка.

4. Оценка степени взаимной зависимости одного параметра от другого и оценка степени влияния каждого параметра на устойчивость геотехнической системы.

5. Определение бифуркационных параметров и оценка значимости параметров в анализе устойчивости геотехнической системы.

6. Выделение ключевых точек контроля и определение контролируемых параметров и методов их регистрации.

7. Оценка стоимости системы геотехнического мониторинга при различных комплектациях (количестве и типах измерительных датчиков)

8. Выбор комплектации системы геотехнического мониторинга по экономическому и техническому критерию.

\section{Результаты практического использования}

Для проверки разработанных подходов были обработаны данные обнаружения суффозионного процесса при геотехническом мониторинге, проводимого в условиях городской застройки в городе Муроме Владимирской области Российской Федерации (координаты $55.557282,42.056503)$ в период с августа 2017 года по май 2018 года. Площадь наблюдения составляла 150х250 метров. В результате обработки данных были выделены ключевые зоны контроля (желтая и зеленая зоны на рис. 1), которые охватывали наиболее уязвимые участки геотехнической системы, а в качестве контролируемых параметров были выбраны электромагнитные параметры грунтов (электрическое сопротивление и диэлектрическая проницаемость среды). В одной из выделенных зон по результатам мониторинговых работ развивался суффозионный процесс (красная зона), который спровоцировал обрушение свода грунта с образованием провала диаметром 4 метра (рис. 2). 


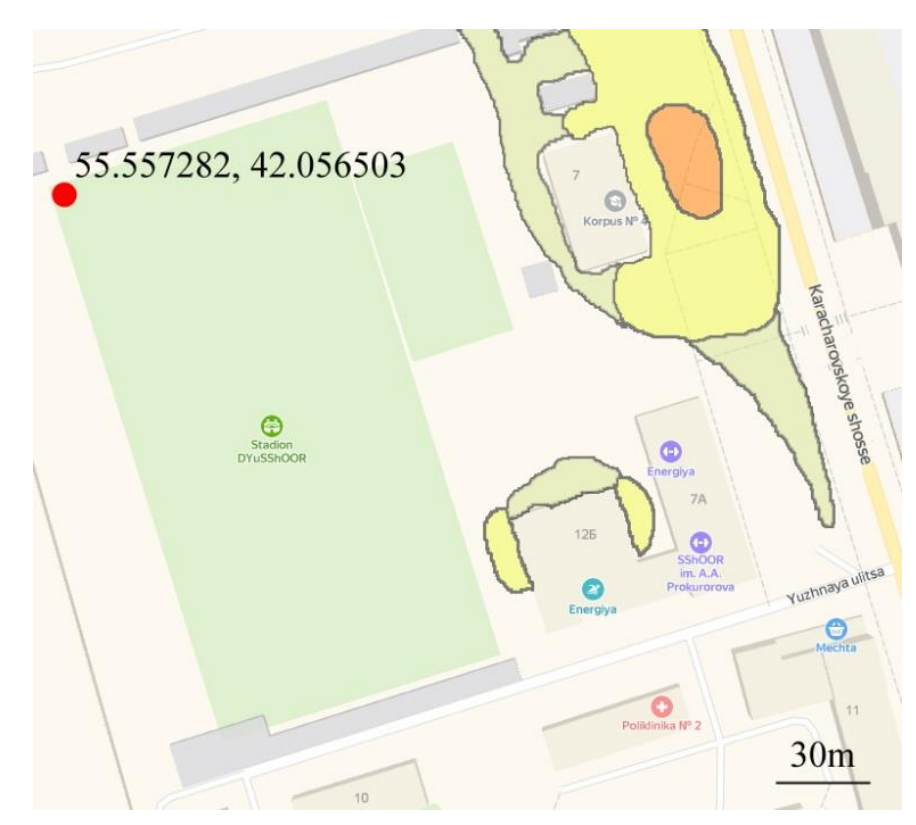

Рис. 1. Выделение ключевых зон контроля

Fig. 1. Allocation of key control areas

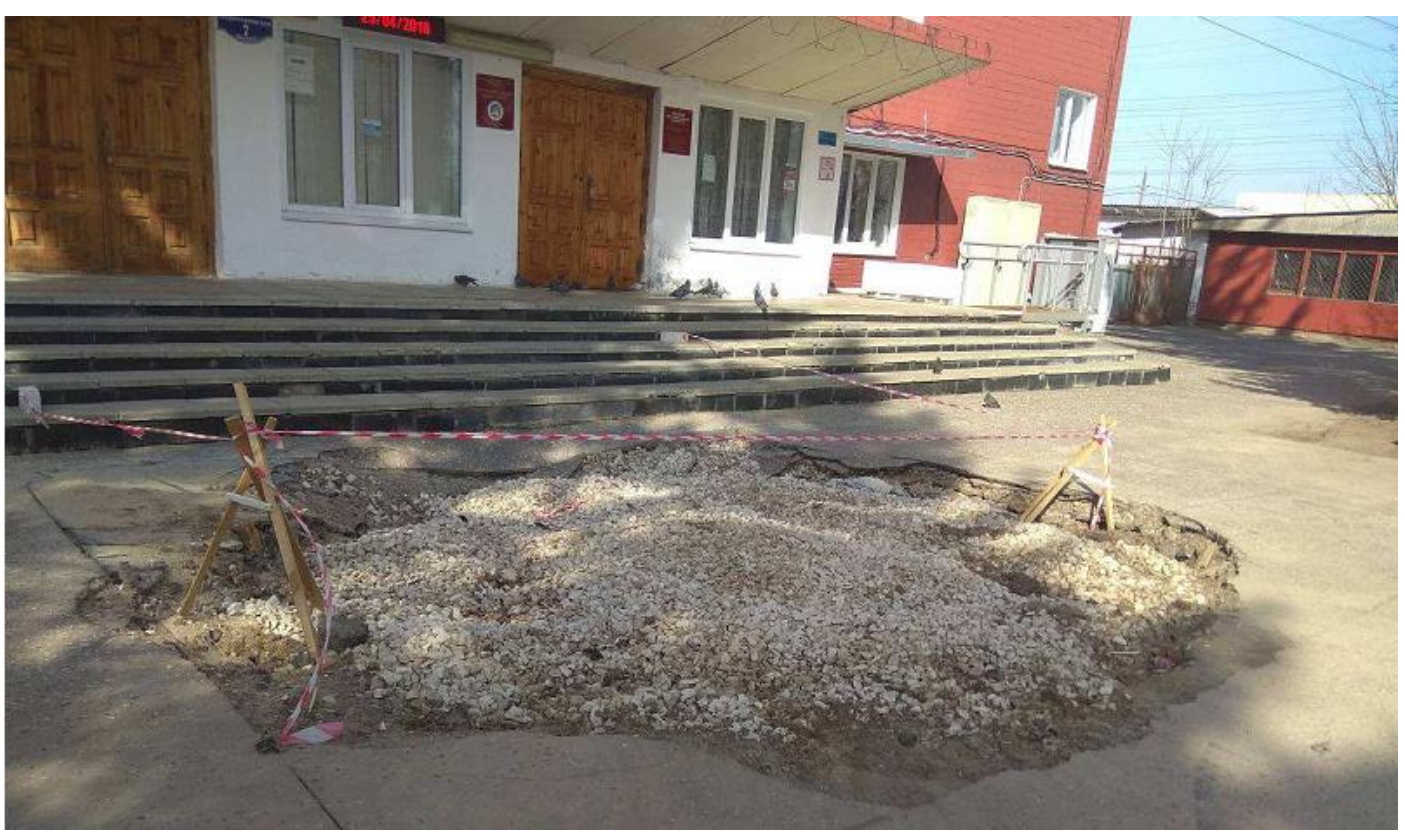

Рис. 2. Провал в ключевой зоне контроля

Fig. 2. Failure in a key zone of control

Система мониторинга выбиралась из следующих представленных систем: георадар типа ОКО-2, система на постоянном токе типа Meduza-48 и фазометрическая система S-1. Формируемые затраты с учетом их стоимости, эксплуатации, вероятности обнаружения и прогнозирования, а так же надежности показы на рис. 3. При этом общий экономический ущерб в геотехнической системе оценивался в 85,7 тысяч долларов. Затраты на проведение мониторинговых работ без предложенного метода регистрации параметров геотехнической системы, который не включает выделение ключевых зон и технико-экономический анализ, и проводится на всей анализируемой площади, приведены на рис. 4. Сравнение произведенных затрат на протяжении 5 лет сведено в табл. 1. 


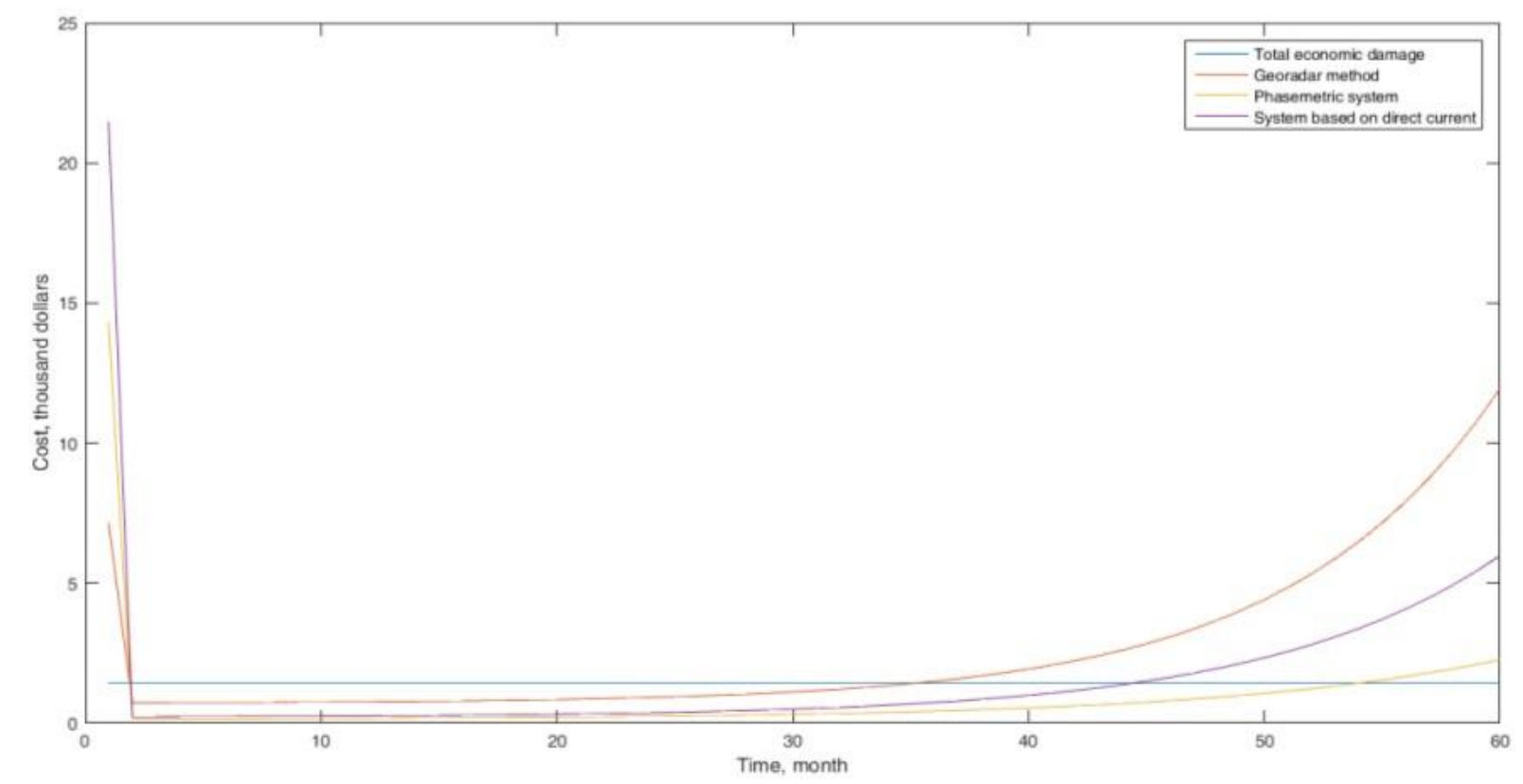

Рис. 3. Сравнение затрат при применении разработанного метода

Fig. 3. Comparison of costs when applying the developed method

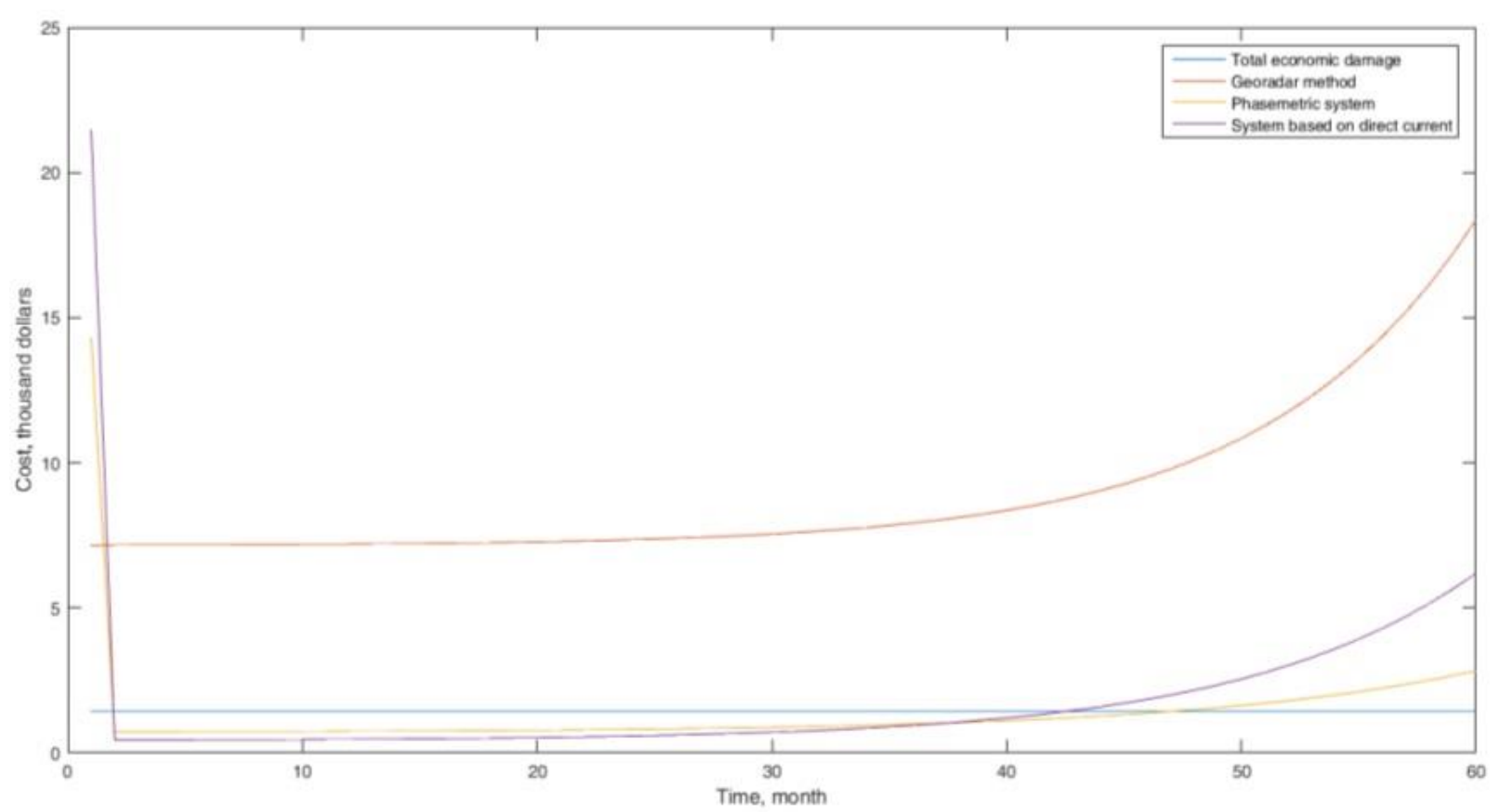

Рис. 4. Сравнение затрат без использования разработанного метода

Fig. 4. Comparison of costs without using the developed method

Таблица 1

Table 1

Сравнение затрат при различных методах измерения за 5 лет

Comparison of costs for different measurement methods over 5 years

\begin{tabular}{|l|l|l|}
\hline \multicolumn{1}{|c|}{ Тип системы мониторинга } & \multicolumn{1}{|c|}{$\begin{array}{c}\text { Без разработанного метода, } \\
\text { тыс. долларов }\end{array}$} & $\begin{array}{c}\text { С разработанным методом, } \\
\text { тыс. долларов }\end{array}$ \\
\hline Георадарное обследование & 535,2 & 155,8 \\
\hline Система на постоянном токе & 107,1 & 94,5 \\
\hline Фазометрическая система & 82,8 & 49,0 \\
\hline
\end{tabular}




\section{Заключение}

Нормативными документами в области строительства и эксплуатации сооружений регламентируется регулярное проведение мониторинговых работ наиболее важных участков геотехнической системы. Однако на практике постоянное проведение таких работ для всех зданий приносит большие издержки и часто игнорируется. Как видно из результатов, описанных в данной статье, разработанный метод обработки параметров геотехнической системы позволяет снизить затраты на проведение мониторинговых работ с одновременным сохранением точности прогнозных оценок, что позволит повысить техногенную безопасность. Для дополнительного снижения затрат возможно построение гибкого графика мониторинга на основе данных о периоде развития деструктивных процессов и комбинировании различных методов измерения. Такой подход хорошо согласуется с задачами и целями геотехнического мониторинга.

\section{Список литературы}

1. ГОСТ Р 53778-2010. Здания и сооружения. Правила обследования и мониторинга технического состояния. Дата введения 25.03.2010.

2. Иноземцев В.К., Иноземцева О.В., Стрельникова К.А. 2010. Расчет бифуркационной устойчивости системы «Сооружение-слой основания» с учетом физической нелинейности основания. Строительство и реконструкция, 1 (27): 16-22.

3. Иноземцев В.К., Редков В.И. 2017. Геотехнические риски строительства и эксплуатации зданий на территориях с оползневыми процессами. Вестник Поволжского отделения Руси. Академия архитектуры и строительных наук, 20: 170-179.

4. Квартальнов С.В., Макулов В.В. 2017. Геотехнический мониторинг зданий и сооружений. European science, 5 (27): 43-45.

5. Леонов О.А., Шкаруба Н.Я. 2012. Алгоритм выбора средств измерений для контроля качества по технико-экономическим критериям. Вестник Федерального государственного образовательного учреждения высшего профессионального образования Московский государственный агроинженерный университет им. В.П. Горячкина, 2 (53): 89-91.

6. Мальцев А.В., Астафьева Н.С., Булавкина Ю.В. 2014. Значение геомониторинга при новом строительстве и реконструкции. Землеустройство и кадастры, 3 (4): 213-218.

7. Назаров Д.И. 2015. Разрушение конструкций горнотехнического здания, энергетический и бифуркационный анализ. Горный информационно-аналитический бюллетень, 7: 95-100.

8. Осокин А.И., Татаринов С.В., Денисова О.О., Макарова Е.В. 2014. Система геотехнического мониторинга как средство обеспечения безопасности строительства. Жилищное строительство, 9: 10-18.

9. Сосунов И.В. 2010. Актуальные вопросы предупреждения чрезвычайных ситуаций, Научнометодическое издание, МЧС России, ФГУ, Рез. Институт гражданской обороны и чрезвычайных ситуаций, 352.

10. Теличенко В.И., Гутенев В.В., Слесарев М.Ю. 2006. Подходы к интерпретации систем управления экологической безопасностью в строительстве. Экология урбанизированных территорий, 2: 6-11.

11. Улицкий В.М., Шашкин А.Г. 1999. Геотехническое сопровождение реконструкции городов: (Обследование, расчеты, ведение работ, мониторинг) . М.: Изд-во АСВ, 324.

12. Bondarik G.K. 2012. Geokibernetika - A tool for diagnosing and predicting the state of natural and natural-technical systems, Geoecol., Eng. geology, Hydrogeol., geocryology, 4: 364-370.

13. Dorofeev N., Kuzichkin O., Eremenko V. 2016. The method of selection of key objects and the construction of forecast function of the destructive geodynamic processes. Informatics, geoinformatics and remote sensing conference proceedings, sgem 2016. Albena, Bulgaria, 1: 883-890.

14. Grecheneva A.V., Kuzichkin O.R., Mikhaleva E.S., Dorofeev N.V. 2018. Geotechnical monitoring of the buildings on the basis of analysis of transfer functions and cyclic vibrational technogenic loads, Jour of Adv Research in Dynamical and Control Systems, Vol. 10, Iss. 02: 1995-2003.

15. Kostarev S. N., Sereda T. G., Mikhailova M.A. 2013. Development of an automated monitoring and management system for natural-technical waste disposal systems, Fund. Res., 6(2): 273-277.

16. Kuzichkin O.R., Grecheneva A.V., Dorofeev N.V., Mishunin V.V. 2018b. Geotechnical monitoring of the objects based on the method of inclinometric control of own frequencies, Journal of Advanced Research in Dynamical and Control Systems, Vol. 10. Iss. 13: 616-619. 
17. Kuzichkin O.R., Grecheneva A.V., Gakhov R.P., Dorofeev N.V., Baknin M.D., Gakhov B.R. 2018a. Development and research of the geoelectric model of the local zone of geodynamic control, Journal of Advanced Research in Dynamical and Control Systems, Vol. 10. Iss. 13: 620-62.

18. Petrochenko V. I., Petrochenko A. V. 2019. Optimization of design solutions for flood protection in river basins, Reclamation, 2 (88): 26-33.

19. Sledge I. J., Keller J. M. 2008. Growing neural gas for temporal clustering. 19th Int. conf. on Pattern Reconition. Tampa, Florida, USA, 1-8.

20. Vasilyev G.S., Kuzichkin O.R., Romanov R.V., Dorofeev N.V., Grecheneva A.V. 2018. The practice of using a multi-pole electrical installation for monitoring the coastal zone of karst lakes. International Multidisciplinary Scientific GeoConference Surveying Geology and Mining Ecology Management, SGEM. 2018 Albena, Bulgaria, Vol. 18. Iss. 1.2: 727-734.

\section{References}

1. GOST R 53778-2010. Buildings and constructions. Rules for inspection and monitoring of technical condition. Date of introduction 25.03.2010. (in Russian)

2. Inozemtsev V. K., Inozemtseva O. V., Strelnikova K. A. 2010. Bifurcational stability of a system "Construction based on a foundation layer with non-linearity of its properties". Construct. and Reconstruct, 1 (27): 16-22. (in Russian)

3. Inozemtsev V.K., Redkov V.I. 2017. Geotechnical Risks of Construction and Operation of Buildings in Landslide Areas. Vestnik Povolzhskogo otdeleniya Rusi. Akademiya arkhitektury i stroitel'nykh nauk, 20: 170-179. (in Russian)

4. Kvartalnov S.V., Makulov V.V. 2017. Geotechnical monitoring of buildings and structures. European science, 5(27): 43-45. (in Russian)

5. Leonov O.A., Shkaruba N.Zh. Selection algorithm of measuring instruments for quality control on technical and economic criteria Vestnik Federal'nogo gosudarstvennogo obrazovatel'nogo uchrezhdeniya vysshego professional'nogo obrazovaniya Moskovskiy gosudarstvennyy agroinzhenernyy universitet im. V.P. Goryachkina, 2 (53): 89-91. (in Russian)

6. Maltsev A. V., Astafyeva N. S., Bulavkina Yu. V. 2014. Value of geomonitoring at new construction and reconstruction. Zemleustroystvo i kadastry, 3(4): 213-218. (in Russian)

7. Nazarov D. I. 2015. The crash of structures of mining engineering buildings, the energy and bifurcation analysis, Mining Inf. and Analytical Bulletin (Sci. and Tech. J.), 7: 95-100. (in Russian)

8. Osokin A.I., Tatarinov S.V., Denisova O.O., Makarova E.V. Geotechnical Monitoring System as a Tool for Ensuring the Safety of Construction. Zhilishchnoye stroitel'stvo, 9: 10-18. (in Russian)

9. Sosunov I. V. 2010. Aktual'nyye voprosy preduprezhdeniya chrezvychaynykh situatsiy, Nauchnometodicheskoye izdaniye [Topical Issues of Prevention of Emergency Situations, Scientific and Methodological Edition] MCHS Rossii, M.: FGU, Rez. Institut grazhdanskoy oborony i chrezvychaynykh situatsiy, 352. (in Russian)

10. Telichenko V.I., Goutenev V.V., Slessarev M. Yu. The approaches to the interpretation of the systems of control over the environmental safety in construction. Ekologiya urbanizirovannykh territoriy, 2: 6-11. (in Russian)

11. Ulitskiy V.M., Shashkin A.G. 1999. Geotekhnicheskoye soprovozhdeniye rekonstruktsii gorodov: (Obsledovaniye, raschety, vedeniye rabot, monitoring). [Geotechnical support of urban reconstruction: (Survey, calculations, work, monitoring)] M.: ASV, 324. (in Russian)

12. Bondarik G. K. 2012. Geokibernetika - A tool for diagnosing and predicting the state of natural and natural-technical systems, Geoecol., Eng. geology, Hydrogeol., geocryology, 4: 364-370.

13. Dorofeev N., Kuzichkin O., Eremenko V. 2016. The method of selection of key objects and the construction of forecast function of the destructive geodynamic processes. Informatics, geoinformatics and remote sensing conference proceedings, sgem 2016. Albena, Bulgaria, 1: 883-890.

14. Grecheneva A.V., Kuzichkin O.R., Mikhaleva E.S., Dorofeev N.V. 2018. Geotechnical monitoring of the buildings on the basis of analysis of transfer functions and cyclic vibrational technogenic loads, Jour of Adv Research in Dynamical and Control Systems, Vol. 10, Iss. 02: 1995-2003.

15. Kostarev S. N., Sereda T. G., Mikhailova M.A. 2013. Development of an automated monitoring and management system for natural-technical waste disposal systems, Fund. Res., 6(2): 273-277.

16. Kuzichkin O.R., Grecheneva A.V., Dorofeev N.V., Mishunin V.V. 2018b. Geotechnical monitoring of the objects based on the method of inclinometric control of own frequencies, Journal of Advanced Research in Dynamical and Control Systems, Vol. 10. Iss. 13: 616-619. 
17. Kuzichkin O.R., Grecheneva A.V., Gakhov R.P., Dorofeev N.V., Baknin M.D., Gakhov B.R. 2018a. Development and research of the geoelectric model of the local zone of geodynamic control, Journal of Advanced Research in Dynamical and Control Systems, Vol. 10. Iss. 13: 620-62.

18. Petrochenko V. I., Petrochenko A. V. 2019. Optimization of design solutions for flood protection in river basins, Reclamation, 2 (88): 26-33.

19. Sledge I. J., Keller J. M. 2008. Growing neural gas for temporal clustering. 19th Int. conf. on Pattern Reconition. Tampa, Florida, USA, 1-8.

20. Vasilyev G.S., Kuzichkin O.R., Romanov R.V., Dorofeev N.V., Grecheneva A.V. 2018. The practice of using a multi-pole electrical installation for monitoring the coastal zone of karst lakes. International Multidisciplinary Scientific GeoConference Surveying Geology and Mining Ecology Management, SGEM. 2018 Albena, Bulgaria, Vol. 18. Iss. 1.2: 727-734.

\section{ИНФОРМАЦИЯ ОБ АВТОРАХ}

\begin{abstract}
Греченева Анастасия Владимировна, кандидат технических наук, доцент кафедры управления и контроля в технических системах Владимирского государственного университета им. Александра Григорьевича и Николая Григорьевича Столетовых, г. Владимир, Россия
\end{abstract}

\begin{abstract}
Дорофеев Николай Викторович, доктор технических наук, доцент, заведующий кафедрой управления и контроля в технических системах Владимирского государственного университета им. Александра Григорьевича и Николая Григорьевича Столетовых, г. Владимир, Россия
\end{abstract}

Панькина Екатерина Сергеевна, научный сотрудник кафедры управления и контроля в технических системах Владимирского государственного университета им. Александра Григорьевича и Николая Григорьевича Столетовых, г. Владимир, Россия

Романов Роман Вячеславович, кандидат технических наук, доцент, доцент кафедры управления и контроля в технических системах Владимирского государственного университета им. Александра Григорьевича и Николая Григорьевича Столетовых, г. Владимир, Россия

\section{INFORMATION ABOUT THE AUTHORS}

Anastasia V. Grecheneva, Candidate of Technical Sciences, Docent of the Department of Management and Control in Technical Systems Vladimir State University, Vladimir, Russia

Nikolay V. Dorofeev, Doctor of Technical Sciences Docent, Head of the Department of Management and Control in Technical Systems Vladimir State University, Vladimir, Russia

Ekaterina S. Pankina, Researcher, Department of Management and Control in Technical Systems Vladimir State University, Vladimir, Russia

Roman V. Romanov, Candidate of Technical Sciences, Docent, Docent of the Department of Management and Control in Technical Systems Vladimir State University, Vladimir, Russia 